Article

\title{
Severity by National Institute of Health Stroke Scale Score and Clinical Features of Stroke Patients with Patent Foramen Ovale Stroke and Atrial Fibrillation
}

\author{
Kaito Abe ${ }^{1,2}\left(\right.$, Fumiya Hasegawa ${ }^{1,2}$, Ryota Nakajima ${ }^{1}$, Hidetoshi Fukui ${ }^{1}$, Moto Shimada ${ }^{1,2}$, \\ Takahiro Miyazaki ${ }^{1,2} \mathbb{D}^{\mathbb{D}}$, Hiroshi Doi ${ }^{1,2}$, Goro Endo ${ }^{1} \mathbb{D}$, Kaori Kanbara ${ }^{1,2}$, Yasuyuki Mochida ${ }^{1,2}$, \\ Jun Okuda ${ }^{1,2}$, Nobuya Maeda ${ }^{3}$, Akira Isoshima ${ }^{4}$, Koichi Tamura ${ }^{2}$ and Tomoaki Ishigami ${ }^{2, *}$
}

check for updates

Citation: Abe, K.; Hasegawa, F.; Nakajima, R.; Fukui, H.; Shimada, M.; Miyazaki, T.; Doi, H.; Endo, G.;

Kanbara, K.; Mochida, Y.; et al. Severity by National Institute of Health Stroke Scale Score and Clinical Features of Stroke Patients with Patent Foramen Ovale Stroke and Atrial Fibrillation. J. Clin. Med. 2021, 10, 332. https://doi.org/10.3390/ jcm10020332

Received: 30 December 2020 Accepted: 14 January 2021 Published: 18 January 2021

Publisher's Note: MDPI stays neutral with regard to jurisdictional claims in published maps and institutional affiliations.

\section{Copyright: (c) 2021 by the authors.} Licensee MDPI, Basel, Switzerland. This article is an open access article distributed under the terms and conditions of the Creative Commons Attribution (CC BY) license (https:// creativecommons.org/licenses/by/ $4.0 /)$.
1 Department of Cardiology, Omori Red Cross Hospital, 4-30-1 Chuo, Ota-Ward, Tokyo 143-8527, Japan; kaitoabemed@gmail.com (K.A.); e103061a@gmail.com (F.H.); cisvjikoryota@gmail.com (R.N.); fukubobu777@yahoo.co.jp (H.F.); petapetapeta@live.jp (M.S.); taka.miyazaki.55@gmail.com (T.M.); doihiroshi6120@yahoo.co.jp (H.D.); goro.08.29.09054261798@docomo.ne.jp (G.E.); kkanbara.circ@gmail.com (K.K.); yasmochi90@gmail.com (Y.M.); jokudajun@yahoo.co.jp (J.O.)

2 Department of Medical Science and Cardiorenal Medicine, Yokohama City University, 3-9 Fukuura Kanazawa-Ward, Yokohama City 236-0004, Japan; tamukou@yokohama-cu.ac.jp

3 Department of Neurology, Omori Red Cross Hospital, 4-30-1 Chuo, Ota-Ward, Tokyo 143-8527, Japan; nobmaeda@khf.biglobe.ne.jp

4 Department of Neurosurgery, Omori Red Cross Hospital, 4-30-1 Chuo, Ota-Ward, Tokyo 143-8527, Japan; a-isoshima@omori.jrc.or.jp

* Correspondence: tommmish@hotmail.com or tommmis@yokohama-cu.ac.jp; Tel.: +81-45-787-2635 (ext. 6312); Fax: +81-45-701-3738

\begin{abstract}
The comparative severity of patent foramen ovale (PFO)-related stroke in patients without atrial fibrillation (AF) and AF-related stroke in patients without PFO is unknown. Therefore, we compared the severity of PFO-related stroke and AF-related stroke. Twenty-six patients who underwent transesophageal echocardiography (TEE) were diagnosed with cardioembolic stroke from July 2018 to March 2020. Cases with AF detected by electrocardiograms or thrombus in the left atrium or left atrial appendage on TEE were included in the AF-related stroke group. Cases with a positive microbubble test on the Valsalva maneuver during TEE, and with no other factors that could cause stroke, were included in the PFO-related stroke group. This study was designed as a single-center, small population pilot study. The stroke severity of the two groups by the National Institute of Health Stroke Scale (NIHSS) score was compared by statistical analysis. Of the 26 cases, five PFO-related stroke patients and 21 AF-related stroke patients were analyzed. The NIHSS score was $2.2 \pm 2.8$ and $11.5 \pm 9.2$ ( $p$-value $<0.01)$, the rate of hypertension was $20.0 \%$ and $85.7 \%(p$-value $=0.01)$, and the $\mathrm{HbA} 1 \mathrm{c}$ value was $5.5 \pm 0.2 \%$ and $6.3 \pm 1.3 \%(p$-value $=0.02)$ in the PFO-related and AF-related stroke groups, respectively. Compared with AF-related stroke patients, stroke severity was low in PFO-related stroke patients.
\end{abstract}

Keywords: patent foramen ovale and stroke; atrial fibrillation and stroke; cryptogenic stroke; severity of stroke; National Institute of Health Stroke Scale score

\section{Introduction}

Stroke results in substantial disability and sometimes causes death [1]. The TOAST classification denotes five subtypes of ischemic stroke: (1) large-artery atherosclerosis, (2) cardioembolism, (3) small-vessel occlusion, (4) stroke of other determined etiology, and (5) stroke of undetermined etiology [2]. Cardioembolic stroke accounts for $15-30 \%$ of ischemic strokes [3].

In up to $40 \%$ of patients with acute ischemic stroke, there is a stroke of undetermined etiology in TOAST classification (5); this stroke has been labeled as cryptogenic 
stroke $[1,2,4,5]$. Major cardioembolic risk sources include atrial fibrillation (AF), recent myocardial infarction, previous myocardial infarction (left ventricular aneurysm), intracardiac thrombus, tumors, rheumatic valve disease, aortic arch atheromatous plaques, endocarditis, and mechanical valve prosthesis, whereas minor or unclear risk sources include patent foramen ovale (PFO), atrial septal aneurysm (ASA), and giant Lambl's excrescences [3]. Evaluation of stroke sources is important for preventing second stroke events.

In the general population, $0.4-1 \%$ have $\mathrm{AF}$, and the prevalence increases to $9 \%$ in the population aged 80 years or older [6]. The CHADS2 and CHA2DS2-VASc risk scores show the frequent occurrence of stroke and embolism, ranging from 0 (low risk) to $18 \%$ event/year (high-risk) among patients with AF [7,8].

For the management of AF, anticoagulant therapy, catheter ablation, and antiarrhythmic drugs are well-established [9]. Recently, transcatheter left atrial appendage closure has been used as a primary therapy for AF patients with contraindications for using chronic oral anticoagulation to prevent stroke [10].

$\mathrm{PFO}$ is caused by incomplete fusion of the septum primum and secundum after birth in the cranial portion of the fossa ovalis, and is a common anatomical variant found in about $25 \%$ of the general population [11,12]. Stroke with PFO occurs when a systemic venous thrombus travels directly into the systemic arterial circulation [1]. The proportion of stroke patients with PFO is 21-63\% [11]. According to a report, cryptogenic stroke patients with PFO were younger and less likely to have conventional vascular risk factors than cryptogenic stroke patients without PFO [11].

Recently, the DEFENSE, REDUCE, and CLOSE trials demonstrated the superiority of PFO closure over medical management [13-15]. In cryptogenic stroke, detection of PFO is important to select an adequate secondary stroke prevention therapy. Transesophageal echocardiography (TEE) is the gold standard for PFO detection. The microbubble test with Valsalva maneuver is recommended for detecting PFO on TEE to avoid the increasing false negative rate of up to $20 \%$ when the Valsalva maneuver is not performed $[3,16]$.

It is known that the severity of ischemic stroke patients with PFO, including patients with $\mathrm{AF}$, is lower than that of patients without PFO, including patients with AF [17]; however, whether the severity of PFO-related stroke in patients without AF is lower than that of AF-related stroke in patients without PFO is unknown. Thus, the purpose of this analysis was to evaluate the severity of PFO-related stroke and AF-related stroke, and to identify the characteristics of both stroke types.

\section{Materials and Methods}

\subsection{Study Design and Patient Population}

We performed a single-center (Omori Red Cross Hospital) retrospective study on consecutive patients with cardioembolic stroke, including suspected cases on magnetic resonance imaging, who underwent TEE between July 2018 and March 2020.

Patients with AF diagnosed from history, electrocardiogram (ECG) at admission, 24 h-holter ECG monitoring, ECG monitoring in the ward, or patients with thrombi including smoke-like echo with a swirling motion of blood in the left atrium (LA) or left atrial appendage (LAA), which is known to be a marker of a prothrombotic state, were classified as AF-related stroke patients [18]. Patients with PFO without AF were classified as PFOrelated stroke patients.

Patients with mobile aortic plaque were defined as Class V in the Katz Index [19], patients after valve replacement and those with cardiac tumor, infectious endocarditis, Lambl's excrescence on the aortic valve, or those diagnosed with atherosclerotic stroke by neurologists after TEE were excluded from this study. Patients with PFO between the right atrium (RA) and LA diagnosed only by the color Doppler method without passage of microbubbles were excluded from this study. 


\subsection{Evaluations}

The diagnosis of ischemic stroke was made by neurologists with known experience in cerebrovascular diseases. TEE was performed and evaluated by cardiologists who were well-experienced in echocardiology. TEE was performed with either Vivid E95 (GE Healthcare, Tokyo, Japan) or ALOKA Prosound $\alpha 10$ (ALOKA, Tokyo, Japan).

\subsection{Baseline Study Assessment}

We collected data on patient characteristics (age, sex, height, body weight, and smoking habit); vascular risk factors; the administration ratio of antiplatelet therapy or oral anticoagulant therapy before stroke onset; blood tests (aspartate transaminase (AST), alanine aminotransferase (ALT), lactate dehydrogenase (LDH), alkaline phosphatase (ALP), total bilirubin (T-bil), brain natriuretic peptide (BNP), hemoglobin, HbA1c, D-dimer, low-density lipoprotein (LDL), high-density lipoprotein (HDL), triglycerides (TGs), creatinine (Cre), estimated glomerular filtration rate (eGFR), and creatinine clearance (CCR) (Cockcroft-Gault equation)); HAS-BLED score to assess bleeding risk in AF patients from hypertension, abnormal renal/liver function, stroke, bleeding history or predisposition, labile INR, elderly ( $>65$ years old), and concomitant drugs/alcohol use [20]; National Institute of Health Stroke Scale (NIHSS) [21]; the risk of paradoxical embolism (RoPE) score to assess the likelihood of the cryptogenic stroke being related to PFO based on the scoring items of age, hypertension, diabetes, history of stroke or transient ischemic attack, smoking habit, and cortical infarct on imaging [11]; ECG; 24 h-holter ECG monitoring; ECG monitoring in the hospital ward; thrombus, including smoke-like echo in LA or LAA by TEE; and left ventricular ejection fraction (LVEF) by transthoracic echocardiography.

The TEE was performed under light sedation with propofol. The LA or LAA thrombi were evaluated in all patients by TEE. For all patients who underwent TEE, an intravenous microbubble test during the Valsalva maneuver was performed. In the GORE-REDUCE trial [14], the classification of PFO size was based on the maximum number of microbubbles during the first three cardiac cycles; 0 microbubbles were classified as no shunt, one to five microbubbles as small, six to 25 microbubbles as moderate, and more than 25 microbubbles as large. PFO was diagnosed by the microbubble test using the Valsalva maneuver technique between the RA and LA. Complex PFO was classified as PFO with ASA, or with a long tunnel length of over $8 \mathrm{~mm}$, or with the eustachian valve [22]. Some AF-related stroke patients with LA or LAA thrombi skipped the microbubble test.

\subsection{Statistical Analysis}

JMP Pro version 15 software (SAS Institute Japan Inc., Tokyo, Japan) was used for statistical analysis. Data are expressed as mean \pm standard deviation for continuous variables and as frequencies and percentages for categorical variables. Baseline characteristics were compared using the Student's $t$ test or Welch's $t$ test for continuous variables and Fisher's exact test for categorical variables. A $p$-value $<0.05$ was considered statistically significant.

\section{Results}

\subsection{Stroke Classification}

A total of 82 patients were enrolled from July 2018 to March 2020. AF-related stroke was noted in $21(25.6 \%)$ patients, atherosclerotic stroke in 21 (25.6\%), PFO-related stroke in five $(6.1 \%)$, cardioembolic stroke without AF in $13(15.9 \%)$, others (systemic lupus erythematosus, hyperemia, vasculitis, lacunar infarction) in four (4.9\%), and cryptogenic stroke in $18(22.0 \%)$ (Figure 1). Cardioembolic stroke without AF included patients with post-valve replacement (four patients), cardiac tumor (two patients), infectious endocarditis (one patient), Lambl's excrescence on the aortic valve (two patients), old myocardial infarction (one patient), and patients with PFO between the RA and LA diagnosed only by the color Doppler method without passage of microbubbles (three patients). 


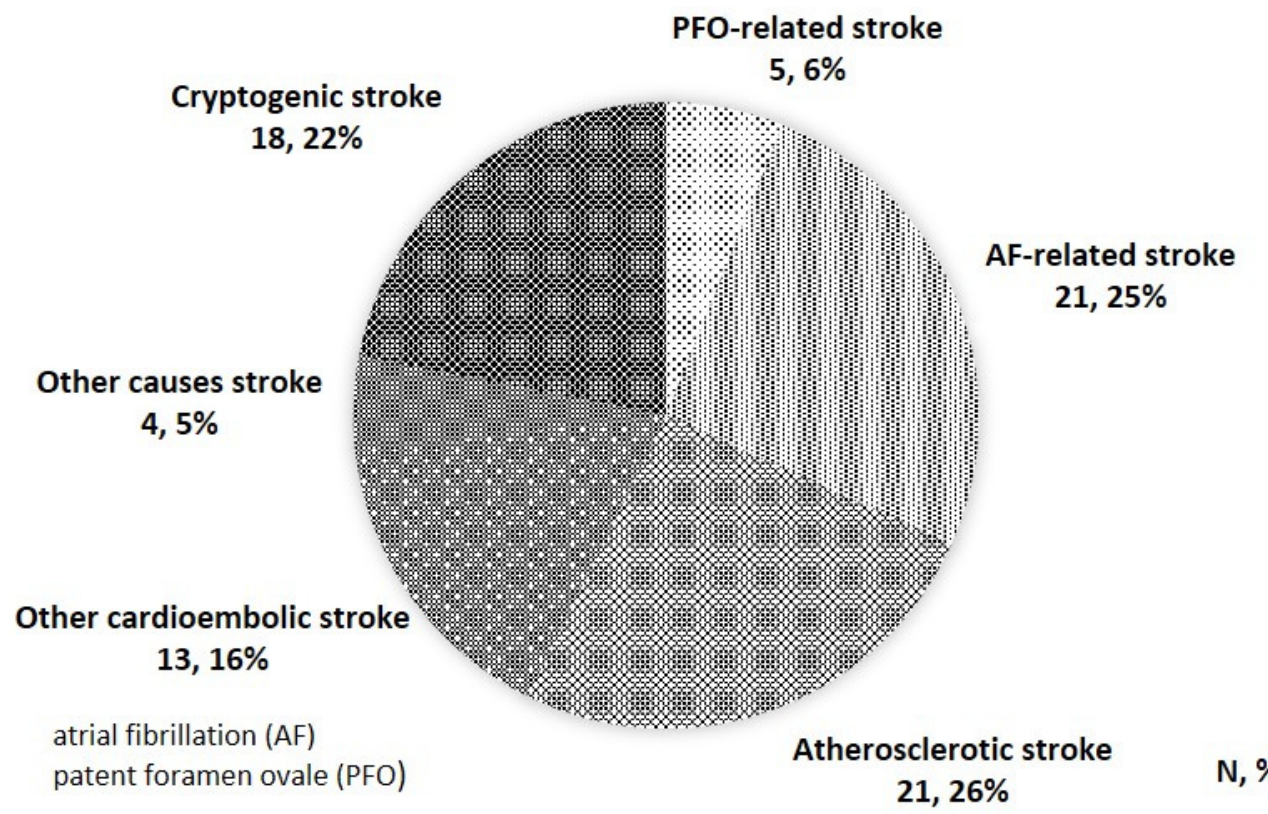

Figure 1. Stroke classification of this study.

\subsection{Study Population and Patient Features}

Among the 21 AF-related stroke patients, 20 (95.2\%) patients had AF and $10(47.6 \%)$ had LA or LAA thrombus, whereas the PFO-related stroke patients had no AF or thrombus in the LA or LAA. The ratio of comorbidity with hypertension in AF-related stroke patients was higher than that in PFO-related stroke patients $(85.7 \%, 20.0 \%, p$-value $=0.01)$. The NIHSS score in AF-related stroke patients was more severe than that in PFO-related stroke patients $(11.5 \pm 9.2,2.2 \pm 2.8, p$-value $<0.01)$. Age, height, body weight, LVEF, and HASBLED score were not significantly different between the groups. The comorbidity ratio of dyslipidemia, diabetes, old myocardial infarction, or past stroke history had no significant differences between the two groups. The administration ratio of antiplatelet therapy or oral anticoagulant therapy before stroke onset had no significant differences between the two groups (Table 1).

Table 1. Baseline patient characteristics of PFO-related stoke and AF-related stroke.

\begin{tabular}{|c|c|c|c|c|}
\hline \multirow{2}{*}{\multicolumn{2}{|c|}{ Characteristics }} & \multirow{2}{*}{$\begin{array}{c}\text { PFO-Related Stroke } \\
(n=5)\end{array}$} & \multirow{2}{*}{$\begin{array}{c}\text { AF-Related Stroke } \\
(n=21)\end{array}$} & \multirow{2}{*}{$p$-Value } \\
\hline & & & & \\
\hline Age & y.o & $58.2 \pm 23.4$ & $77.9 \pm 7.6$ & n.s * \\
\hline Sex (Male) & $n(\%)$ & $4(80 \%)$ & $14(66.7 \%)$ & n.s $\dagger$ \\
\hline Height & $\mathrm{cm}$ & $166.4 \pm 7.3$ & $162.0 \pm 8.0$ & n.s \\
\hline Body Weight & $\mathrm{kg}$ & $59.9 \pm 10.7$ & $58.9 \pm 11.0$ & n.s \\
\hline Smoking Habit & $n(\%)$ & $5(100 \%)$ & $11(52.4 \%)$ & n.s + \\
\hline Hypertension & $n(\%)$ & $1(20 \%)$ & $18(85.7 \%)$ & $0.01+$ \\
\hline Dyslipidemia & $n(\%)$ & $3(60 \%)$ & $11(52.4 \%)$ & n.s $†$ \\
\hline Diabetes & $n(\%)$ & $0(0 \%)$ & $7(33.3 \%)$ & n.s $†$ \\
\hline OMI & $n(\%)$ & $0(0 \%)$ & $2(9.5 \%)$ & n.s + \\
\hline History of Stroke & $n(\%)$ & $0(0 \%)$ & $2(9.5 \%)$ & n.s + \\
\hline PFO & $n(\%)$ & $5(100 \%)$ & $0(0 \%)$ & $<0.01+$ \\
\hline $\mathrm{AF}$ & $n(\%)$ & $0(0 \%)$ & $20(95.2 \%)$ & $<0.01+$ \\
\hline LA/LAA thrombus & $n(\%)$ & $0(0 \%)$ & $10(47.6 \%)$ & n.s + \\
\hline LVEF & $\%$ & $69.0 \pm 5.2$ & $66.0 \pm 8.4$ & n.s \\
\hline
\end{tabular}


Table 1. Cont.

\begin{tabular}{ccccc}
\hline \multirow{2}{*}{ Characteristics } & & PFO-Related Stroke & AF-Related Stroke & \\
\cline { 3 - 4 } & & $(\boldsymbol{n}=\mathbf{5})$ & $\mathbf{( n = 2 1 )}$ & \\
\hline Antiplatet Therapy & $n(\%)$ & $2(40 \%)$ & $7(33.3 \%)$ & n.s + \\
Anticoagulant Therapy & $n(\%)$ & $0(0 \%)$ & $4(19 \%)$ & \\
DOAC & $n(\%)$ & & $3(14.3 \%)$ & \\
Warfarin & $n(\%)$ & & $1(4.8 \%)$ & n.s \\
HAS-BLED Score & & $2.2 \pm 1.6$ & $3.3 \pm 0.9$ & $<0.01$ * \\
NIHSS Score & & $2.2 \pm 2.8$ & $11.5 \pm 9.2$ & \\
\hline
\end{tabular}

Atrial fibrillation (AF), direct oral anticoagulants (DOAC), left atrium (LA), left atrial appendage (LAA), left ventricular ejection fraction (LVEF), National Institute of Health Stroke Scale (NIHSS), old myocardial infarction (OMI), patent foramen ovale (PFO), years old (y.o); ${ }^{*}$ Welch's $t$ test, + Fisher's exact test.

$\mathrm{HbA} 1 \mathrm{c}$ values in AF-related stroke patients were higher than that in PFO-related stroke patients $(6.3 \% \pm 1.3 \%, 5.5 \% \pm 0.2 \%$, $p$-value $=0.02)$. BNP, D-dimer, hemoglobin, LDL, HDL, TG, Cre, eGFR, CCR, AST, ALT, T-bil, and ALP were not significantly different between the groups (Table 2).

Table 2. Baseline patient blood test parameters of PFO-related stoke and AF-related stroke.

\begin{tabular}{|c|c|c|c|c|}
\hline \multirow{2}{*}{\multicolumn{2}{|c|}{ Blood Test Parameters }} & \multirow{3}{*}{$\begin{array}{c}\text { PFO-Related Stroke } \\
(n=5) \\
39.5 \pm 28.1\end{array}$} & \multirow{3}{*}{$\begin{array}{c}\text { AF-Related Stroke } \\
(\boldsymbol{n}=\mathbf{2 1}) \\
155.4 \pm 189.0\end{array}$} & \multirow{3}{*}{$\frac{p \text {-Value }}{\text { n.s } *}$} \\
\hline & & & & \\
\hline $\mathrm{BNP}$ & $\mathrm{pg} / \mathrm{mL}$ & & & \\
\hline D-dimer & $\mathrm{ug} / \mathrm{mL}$ & $3.7 \pm 5.6$ & $2.0 \pm 2.4$ & n.s * \\
\hline $\mathrm{Hb}$ & $\mathrm{g} / \mathrm{dL}$ & $14.4 \pm 1.3$ & $13.8 \pm 2.0$ & n.s \\
\hline $\mathrm{HbA1c}$ & $\%$ & $5.5 \pm 0.2$ & $6.3 \pm 1.3$ & $0.02 *$ \\
\hline LDL & $\mathrm{mg} / \mathrm{dL}$ & $116.0 \pm 32.0$ & $110.3 \pm 42.4$ & n.s \\
\hline HDL & $\mathrm{mg} / \mathrm{dL}$ & $67.2 \pm 25.6$ & $57.5 \pm 17.4$ & n.s \\
\hline TG & $\mathrm{mg} / \mathrm{dL}$ & $112.6 \pm 55.6$ & $125.1 \pm 98.7$ & n.s \\
\hline Cre & $\mathrm{mg} / \mathrm{dL}$ & $0.84 \pm 0.2$ & $1.01 \pm 0.5$ & n.s \\
\hline eGFR & $\mathrm{ml} / \mathrm{min} / 1.73 \mathrm{~m} 2$ & $73.8 \pm 17.4$ & $58.9 \pm 20.6$ & n.s \\
\hline CCR & $\mathrm{ml} / \mathrm{min}$ & $83.2 \pm 35.5$ & $56.5 \pm 23.7$ & n.s \\
\hline AST & $\mathrm{IU} / 1$ & $19.0 \pm 4.7$ & $23.4 \pm 5.8$ & n.s \\
\hline ALT & $\mathrm{IU} / 1$ & $15.6 \pm 2.7$ & $15.5 \pm 6.4$ & n.s \\
\hline $\mathrm{LDH}$ & $\mathrm{IU} / 1$ & $178.0 \pm 18.4$ & $231.0 \pm 45.2$ & 0.02 \\
\hline T-bil & $\mathrm{mg} / \mathrm{dL}$ & $0.62 \pm 0.23$ & $0.77 \pm 0.32$ & n.s \\
\hline ALP & $\mathrm{IU} / 1$ & $182.4 \pm 70.8$ & $237.4 \pm 95.3$ & n.s \\
\hline
\end{tabular}

Atrial fibrillation (AF), alkaline phosphatase (ALP), alanine aminotransferase (ALT), aspartate transaminase (AST), brain natriuretic peptide (BNP), creatinine clearance (CCR) (Cockcroft-Gault equation), creatinine (Cre), estimated glomerular filtration rate (eGFR), hemoglobin $(\mathrm{Hb})$, high-density lipoprotein (HDL), lactate dehydrogenase (LDH), low-density lipoprotein (LDL), patent foramen ovale (PFO), total bilirubin (T-bil), triglycerides (TG); ${ }^{*}$ Welch's $t$ test.

\subsection{PFO Characteristics of This Study}

Among the five PFO-related stroke patients, one patient had a small shunt PFO, two patients had moderate shunt PFO, and two patients had a large shunt PFO. Of the five PFO-related stroke patients, four patients had complex PFO; all complex PFOs had a long PFO tunnel, and had no eustachian valve or ASA. The RoPE score was $4.8 \pm 2.4$, the average PFO attributable fraction was $36.0 \pm 37.3 \%$, and the estimated two years stroke recurrence rate was $12.2 \pm 7.2 \%$ in this PFO population by RoPE score to assess the likelihood of the cryptogenic stroke being related to PFO (Table 3) [11]. 
Table 3. PFO characteristics, PFO attributable fraction, and estimated two years stroke recurrence rate by RoPE score.

\begin{tabular}{|c|c|c|}
\hline \multirow{2}{*}{ PFO Characteristics } & & \multirow{2}{*}{$\begin{array}{c}\text { PFO-Related Stroke } \\
(n=5)\end{array}$} \\
\hline & & \\
\hline PFO Size & & \\
\hline Small & $n$ & 1 \\
\hline Moderate & $n$ & 2 \\
\hline Large & $n$ & 2 \\
\hline Rope Score & & $4.8 \pm 2.4$ \\
\hline PFO-Attributable Fraction & $\%$ & $36.0 \pm 37.3$ \\
\hline Estimated Two Years Stroke Recurrence Rate & $\%$ & $12.2 \pm 7.2$ \\
\hline
\end{tabular}

\section{Discussion}

The novelty of this study is that it is a PFO group diagnosed by TEE with the highly diagnostic Valsalva maneuver technique, and that it includes the severity of stroke between PFO-related stroke in patients without AF and AF-related stroke in patients without PFO.

Severity comparison by the NIHSS showed that AF-related stroke was more severe than PFO-related stroke. Previous studies [17] also reported that PFO-related stroke was less severe than strokes in the other groups; it was expected that AF-related stroke would be more severe, which was also revealed in our study. Regarding administration of oral medication before stroke onset, antiplatelet therapy was administered to two $(40 \%)$ patients in the PFO-related stroke group. In the AF-related stroke group, anticoagulation therapy was administered to four (19.0\%) patients and antiplatelet therapy to seven (33.3\%). It may be necessary to consider the effect of these pre-medications on the severity of stroke.

Generally, it is said that PFO-related stroke patients are younger and have less cardiovascular risk; this study also showed that the ratio of comorbidity of hypertension and high $\mathrm{HbA1c}$ in blood tests was significantly lower in the PFO-related stroke patients compared to AF-related stroke patients. Although there was no significant difference in other factors, the age, ratio of comorbidity of dyslipidemia, diabetes, and ratio of smoking habits tended to be low in the PFO-related stroke group, which was similar to the existing report [11]. In particular, the average age of the PFO-related stroke patients was 58.2 years, which is younger than that of the AF-related stroke patients (77.9 years), and it is necessary to consider that the fact that there are few cardiovascular risk factors may also contribute to the low severity of stroke in the PFO-related stroke patients. For younger patients, functional improvement after stroke can be expected, and observation of long prognosis is also important.

It has been reported that the probability of stroke due to PFO is $88 \%$, and the recurrence rate after two years is $2 \%$ in the group with the highest RoPE Score [11]. The PFO attributable stroke rate in this study is not high at $36.0 \%$, but the average recurrence probability after two years by the RoPE score is estimated to be high at $12.2 \%$. Therefore, secondary prevention therapies, such as antiplatelet therapy, anticoagulant therapy, or a PFO occluder device, seemed to be important in decreasing the recurrence rate of strokes.

This study has some limitations. This study is a pilot study not registered in clinical trials with an international clinical trials register. As this was a retrospective analysis at a single institution, it is necessary to consider the influence of the small population, five PFO-related stroke patients and 21 AF-related stroke patients. Statistical results may not be sufficient, as power analysis has not been performed in this study. It is also necessary to consider the influence of population bias, such as pre-test probability, because it is a group of cases in which neurologists suspected cardioembolic stroke and who needed TEE. In addition, there is a possibility that latent AF and PFO may be involved in cases of stroke other than cardioembolic stroke diagnosed by neurologists. Since patient prognosis 
was not observed in this study, it is necessary to observe their prognosis after medical interventions. We need to be careful in interpreting the results of this study.

There were $18(22.0 \%)$ cases in the stroke group with unknown causes in the analysis target, and four of them were suspected of being cardioembolic stroke by the neurologist, but definitive clinical findings were unclear. It seems necessary to evaluate the comorbidity of AF with an implantable electrocardiograph.

In this analysis, TEE with the Valsalva maneuver technique was performed for PFO detection; therefore, PFO detection in this study was highly credible. However, it is said that there are detection limits of this method; hence, it seems that there is a possibility of wrongly classifying other strokes into the cryptogenic stroke group.

In addition, even in the group diagnosed with cryptogenic stroke, the detection rate of thrombus in LA/LAA may have decreased, because at the time of TEE, antiplatelet therapy or anticoagulant therapy had already been performed. In this study, after stroke, anticoagulant therapy was administered to three patients, and antiplatelet therapy was administered to one patient in the PFO-related stroke group. In the AF-related stroke group, anticoagulant therapy was administered to all patients. Of these, anticoagulation therapy alone was administered to $19(90.5 \%)$ patients, and a combination of antiplatelet therapy and anticoagulant therapy was administered to two (9.5\%) patients. Therefore, it is necessary to observe the secondary preventive effect on the recurrence rate in the future.

\section{Conclusions}

Compared with AF-related stroke patients, stroke severity, the comorbidity of hypertension rate, and the value of $\mathrm{HbA1c}$ were low in PFO-related stroke patients.

Author Contributions: Conceptualization, K.A.; methodology, K.A.; software, K.A. and T.I.; validation, K.A., F.H., R.N., H.F., M.S., T.M., H.D., G.E., K.K., Y.M., J.O., N.M., A.I., K.T. and T.I.; formal analysis, K.A. and T.I.; investigation, K.A. and T.I.; resources, K.A., F.H., R.N., H.F., M.S., T.M., H.D., G.E., K.K., Y.M., J.O., N.M. and A.I.; data curation, K.A. and T.I.; writing-original draft preparation, K.A.; writing-review and editing, K.T. and T.I.; visualization, K.A. and T.I.; supervision, N.M., A.I., K.T. and T.I.; project administration, K.A. and T.I.; All authors have read and agreed to the published version of the manuscript.

Funding: This research received no external funding.

Institutional Review Board Statement: The study was conducted according to the guidelines of the Declaration of Helsinki, and approved by the ethical committee of Omori Red Cross Hospital (protocol code: No.20-5, 26 May 2020).

Informed Consent Statement: Informed consent was obtained from all subjects involved in the study.

Data Availability Statement: The data presented in this study are available on request from the corresponding author.

Acknowledgments: We are grateful to departments of neurology, neurosurgery, and cardiology staffs for stroke patients' care.

Conflicts of Interest: The authors declare no conflict of interest.

\section{References}

1. Mir, H.; Siemieniuk, R.A.C.; Ge, L.C.; Foroutan, F.; Fralick, M.; Syed, T.; Lopes, L.C.; Kuijpers, T.; Mas, J.-L.; Vandvik, P.O.; et al. Patent foramen ovale closure, antiplatelet therapy or anticoagulation in patients with patent foramen ovale and cryptogenic stroke: A systematic review and network meta-analysis incorporating complementary external evidence. BMJ Open 2018, 8, e023761. [CrossRef] [PubMed]

2. Adams, H.P.; Bendixen, B.H.; Kappelle, L.J.; Biller, J.; Love, B.B.; Gordon, D.L.; Marsh, E.E. Classification of subtype of acute ischemic stroke. Definitions for use in a multicenter clinical trial. TOAST. Trial of Org 10172 in Acute Stroke Treatment. Stroke 1993, 24, 35-41. [CrossRef] [PubMed]

3. Pepi, M.; Evangelista, A.; Nihoyannopoulos, P.; Flachskampf, F.A.; Athanassopoulos, G.; Colonna, P.; Habib, G.; Ringelstein, E.B.; Sicari, R.; Zamorano, J.L.; et al. Recommendations for echocardiography use in the diagnosis and management of cardiac sources of embolism: European Association of Echocardiography (EAE) (a registered branch of the ESC). Eur. J. Echocardiogr. 2010, 11, 461-476. [CrossRef] 
4. Osteraas, N.D.; Vargas, A.; Cherian, L.; Song, S. Role of PFO Closure in Ischemic Stroke Prevention. Curr. Treat. Options Cardiovasc. Med. 2019, 21, 63. [CrossRef]

5. Benjamin, E.J.; Blaha, M.J.; Chiuve, S.E.; Cushman, M.; Das, S.R.; Deo, R.; de Ferranti, S.D.; Floyd, J.; Fornage, M.; Gillespie, C.; et al. Heart Disease and Stroke Statistics-2017 Update: A Report From the American Heart Association. Circulation 2017, 135, e146-e603. [CrossRef] [PubMed]

6. Go, A.S.; Hylek, E.M.; Phillips, K.A.; Chang, Y.; Henault, L.E.; Selby, J.V.; Singer, D.E. Prevalence of diagnosed atrial fibrillation in adults: National implications for rhythm management and stroke prevention: The AnTicoagulation and Risk Factors in Atrial Fibrillation (ATRIA) Study. JAMA 2001, 285, 2370-2375. [CrossRef]

7. Gage, B.F.; Waterman, A.D.; Shannon, W.; Boechler, M.; Rich, M.W.; Radford, M.J. Validation of clinical classification schemes for predicting stroke: Results from the National Registry of Atrial Fibrillation. JAMA 2001, 285, 2864-2870. [CrossRef]

8. Lip, G.Y.H.; Nieuwlaat, R.; Pisters, R.; Lane, D.A.; Crijns, H.J.G.M. Refining clinical risk stratification for predicting stroke and thromboembolism in atrial fibrillation using a novel risk factor-based approach: The euro heart survey on atrial fibrillation. Chest 2010, 137, 263-272. [CrossRef]

9. Ono, K.; Iwasaki, Y.; Shimizu, W.; Akao, M.; Ikeda, T.; Ishii, K.; Inden, Y.; Kusano, K.; Kobayashi, Y.; Koretsune, Y.; et al. JCS/JHRS 2020 Guideline on Pharmacotherapy of Cardiac Arrhythmias. Available online: https://www.j-circ.or.jp/cms/wp-content/ uploads/2020/01/JCS2020_Ono.pdf (accessed on 28 August 2020).

10. Majule, D.N.; Jing, C.; Rutahoile, W.M.; Shonyela, F.S. The Efficacy and Safety of the WATCHMAN Device in LAA Occlusion in Patients with Non-Valvular Atrial Fibrillation Contraindicated to Oral Anticoagulation: A Focused Review. Ann. Thorac. Cardiovasc. Surg. 2018, 24, 271-278. [CrossRef]

11. Kent, D.M.; Ruthazer, R.; Weimar, C.; Mas, J.-L.; Serena, J.; Homma, S.; Di Angelantonio, E.; Di Tullio, M.R.; Lutz, J.S.; Elkind, M.S.V.; et al. An index to identify stroke-related vs incidental patent foramen ovale in cryptogenic stroke. Neurology 2013, 81, 619-625. [CrossRef]

12. Homma, S.; Sacco, R.L. Patent foramen ovale and stroke. Circulation 2005, 112, 1063-1072. [CrossRef] [PubMed]

13. Lee, P.H.; Song, J.-K.; Kim, J.S.; Heo, R.; Lee, S.; Kim, D.-H.; Song, J.-M.; Kang, D.-H.; Kwon, S.U.; Kang, D.-W.; et al. Cryptogenic Stroke and High-Risk Patent Foramen Ovale: The DEFENSE-PFO Trial. J. Am. Coll. Cardiol. 2018, 71, 2335-2342. [CrossRef] [PubMed]

14. Søndergaard, L.; Kasner, S.E.; Rhodes, J.F.; Andersen, G.; Iversen, H.K.; Nielsen-Kudsk, J.E.; Settergren, M.; Sjöstrand, C.; Roine, R.O.; Hildick-Smith, D.; et al. Patent Foramen Ovale Closure or Antiplatelet Therapy for Cryptogenic Stroke. N. Engl. J. Med. 2017, 377, 1033-1042. [CrossRef] [PubMed]

15. Mas, J.-L.; Derumeaux, G.; Guillon, B.; Massardier, E.; Hosseini, H.; Mechtouff, L.; Arquizan, C.; Béjot, Y.; Vuillier, F.; Detante, O.; et al. Patent Foramen Ovale Closure or Anticoagulation vs. Antiplatelets after Stroke. N. Engl. J. Med. 2017, 377, $1011-1021$. [CrossRef] [PubMed]

16. Rodrigues, A.C.; Picard, M.H.; Carbone, A.; Arruda, A.L.; Flores, T.; Klohn, J.; Furtado, M.; Lira-Filho, E.B.; Cerri, G.G.; Andrade, J.L. Importance of adequately performed Valsalva maneuver to detect patent foramen ovale during transesophageal echocardiography. J. Am. Soc. Echocardiogr. 2013, 26, 1337-1343. [CrossRef]

17. Consoli, D.; Paciaroni, M.; Galati, F.; Aguggia, M.; Melis, M.; Malferrari, G.; Consoli, A.; Vidale, S.; Bosco, D.; Cerrato, P.; et al. Prevalence of Patent Foramen Ovale in Ischaemic Stroke in Italy: Results of SISIFO Study. Cerebrovasc. Dis. 2015, 39, 162-169. [CrossRef]

18. Sahin, O.; Savas, G. Relationship between presence of spontaneous echo contrast and platelet-to-lymphocyte ratio in patients with mitral stenosis. Echocardiography 2019, 36, 924-929. [CrossRef]

19. Katz, E.S.; Tunick, P.A.; Rusinek, H.; Ribakove, G.; Spencer, F.C.; Kronzon, I. Protruding aortic atheromas predict stroke in elderly patients undergoing cardiopulmonary bypass: Experience with intraoperative transesophageal echocardiography. J. Am. Coll. Cardiol. 1992, 20, 70-77. [CrossRef]

20. Pisters, R.; Lane, D.A.; Nieuwlaat, R.; De Vos, C.B.; Crijns, H.J.G.M.; Lip, G.Y.H. A Novel User-Friendly Score (HAS-BLED) To Assess 1-Year Risk of Major Bleeding in Patients with Atrial Fibrillation. Chest 2010, 138, 1093-1100. [CrossRef]

21. Brott, T.; Adams, H.P.; Olinger, C.P.; Marler, J.R.; Barsan, W.G.; Biller, J.; Spilker, J.; Holleran, R.; Eberle, R.; Hertzberg, V. Measurements of acute cerebral infarction: A clinical examination scale. Stroke 1989, 20, 864-870. [CrossRef]

22. Vitarelli, A.; Mangieri, E.; Capotosto, L.; Tanzilli, G.; D’Angeli, I.; Toni, D.; Azzano, A.; Ricci, S.; Placanica, A.; Rinaldi, E.; et al. Echocardiographic findings in simple and complex patent foramen ovale before and after transcatheter closure. Eur. Heart $J$. Cardiovasc. Imaging 2014, 15, 1377-1385. [CrossRef] [PubMed] 